\title{
Transcriptome-Based Analysis of Euglena gracilis Lipid Metabolic Pathways Under Light Stress
}

\author{
Wen-hui Zhang ${ }^{1,2}$, Jinwei Gao ${ }^{1,3}$, Wenli Zhou ${ }^{1, *}\left(\mathbb{D}\right.$, Yeong Yik Sung ${ }^{2}$
}

\author{
${ }^{1}$ Tianjin Agricultural University, Tianjin Key Laboratory of Aqua-Ecology \& Aquaculture, Fisheries College, Tianjin, \\ China, 300384 \\ 2 Universiti Malaysia Terengganu, Institute of Marine Biotechnology (IMB), 21030, Kuala Terengganu, Terengganu, \\ Malaysia; \\ ${ }^{3}$ Quality of Fishery Environment and Aquatic Products Supervision and Testing Center (Tianjin), Ministry of Agriculture \\ and Village, 300221, Tianjin, China.
}

\section{Article History}

Received 22 April 2019

Accepted 07 November 2019

First Online 25 November 2019

\section{Corresponding Author}

Tel.: +8615122401092

E-mail:wlzhou69@126.com

\section{Keywords}

Euglena gracilis

Amino acids

Transcriptome

Lipid metabolism

\begin{abstract}
To investigate lipid metabolism pathways and the genes encoding essential enzymes regulating these pathways, the present study was conducted to analyze the transcriptome of Euglena gracilis cultured under light and dark conditions for 5 days using the Illumina HiSeq 4000 sequencing platform. A total of 120,086 unigenes were obtained using Trinity assembly software, of which 48,031 were annotated. Through bioinformatics analysis, genes associated with E. gracilis lipid metabolism pathways were identified, and pathways for fatty acid biosynthesis, fatty acid degradation, and glycerolipid metabolism were preliminarily constructed. In addition, differences in gene expression under different culture conditions were compared. By regulating the activity of acetyl-CoA carboxylase, 3-ketoacyl-ACP synthase II, and phospholipid: diacylglycerol acyltransferase, enzymes associated with fatty acid and glycerolipid degradation may be inhibited or knocked out, thereby increasing fatty acid and glycerolipid content in E. gracilis. This study provides a basis for genetic modification of $E$. gracilis for construction of a high-quality oil-producing engineered strain.
\end{abstract}

\section{Introduction}

With globalization and the rapid development of the global economy, the energy crisis has become increasingly prominent (Molony, 2011). Massive exploitation and overdevelopment of traditional fossil fuels has led to an acute shortage of resources and supplies (Coyle \& Simmons, 2014). Because of environmental degradation and petroleum depletion resulting from unreasonable energy consumption, biodiesel is receiving increasing attention. One source of biodiesel, namely the many species of microalgae, accumulate triacylglycerols (TAG) during growth and photosynthesis (Hu et al., 2010). After microalgae are used to prepare biodiesel, the residual biomass can be converted into valuable industrial materials (Krajčovič, Vesteg, \& Schwartzbach, 2015). The lipids synthesized by microalgal cells constitute a highly promising source of raw material for biodiesel production. In the 1970s, the United States National Renewable Energy Laboratory led the pioneering Aquatic Species ProgramBiodiesel from Algae (ASP) program. In the subsequent years (Savage, 2011; Ho, Chen, Lee, \& Chang, 2010), microalgal biodiesel production has gradually become a global research focus (Scott et al., 2010; Yusuf, 2007).

Euglena gracilis is a single-celled eukaryotic species found in fresh water. It has a facultative mode of nutrition (Ahmadinejad, Dagan, \& Martin, 2007), as it can produce nutrients not only through photosynthesis (Pulz \& Gross, 2004), but also by ingesting organic substances such as beef extract, peptone, acetate, and ethanol through osmotic nutrition (Afiukwa \& Ogbonna, 2017; Ogbonna, 2009). This "plant-animal duality" makes it rich in nutrients such as unsaturated fatty acids, polysaccharides, vitamins, and amino acids. 
Light intensity not only directly affected the growth of microalgae, but also affected the pigment and ester contents of microalgae (Khozin-Goldberg, Bigogno, Shrestha, \& Cohen, 2010; Solovchenko, KhozinGoldberg, Didi-Cohen, Cohen, \& Merzlyak, 2008b; Solovchenko, Khozin-Goldberg, Didi-Cohen, Cohen, \& Merzlyak, 2008a). Solovchenko, Khozin-Goldberg, DidiCohen, Cohen, \& Merzlyak, 's research (2008b) found that high light intensity was beneficial to increase the biomass of Parietochloris incisa and for accumulation of oil, but AA content was the highest under medium light intensity. The results of Zhang's study (Zhang, Cohen, Khozin-Goldberg, \& Richmond, 2002) showed that higher intensity light conditions could rapidly promote the growth of Parietochloris incisa, but the AA content was not high. However, Sun, Hasan, Hobba, Nevalainen, \& Te'o (2018) showed the highest accumulation of paramylose was the largest in $E$. gracilis under dark conditions.

Currently, methods such as genetic engineering, genetic manipulation, and metabolic regulation are used to construct engineered algal strains, purposefully regulate gene expression, obtain dominant algal strains, and induce accumulation of large amounts of fatty acids in microalgae by regulating essential enzymes in fatty acid biosynthesis (Rawisara, Supapon, \& Kobkul, 2009). For example, heterologous expression of enzymes associated with TAG biosynthesis induces the accumulation of large amounts of TAG in Chlorella (Hsieh, Su, \& Chien, 2012). However, not all essential enzymes can be overexpressed. High expression of 3ketoacyl-acyl carrier protein synthase III (KASIII) in tobacco, for instance, increases palmitic acid content but reduces the rate of fatty acid synthesis (Dehesh, Tai, Edwards, Byrne, \& Jaworski, 2001). For effective engineered regulation of fatty acid biosynthesis in $E$. gracilis, it is therefore necessary to first perform indepth data-mining, build a core metabolic network, and analyze its regulatory mechanisms.

In this study, the content and composition of fatty acids in $E$. gracilis were analyzed under light stress conditions. The Illumina HiSeq 4000 sequencing platform was used for sequencing an $E$. gracilis transcriptome library, and a large amount of genetic data was obtained. Functional annotation and pathway analysis of the unigenes from the assembled sequences was performed. Through analysis of transcriptome data, genes essential for fatty acid biosynthesis, fatty acid degradation, and triacylglycerol metabolism in E. gracilis were studied, and their metabolic pathways constructed. Differential pathway-associated genes in the experimental group were analyzed with the goal of using genetic engineering technology to construct a high-quality engineered algal strain as a candidate and theoretical basis for large-scale, sustainable biodiesel production.

\section{Materials and Methods}

\section{Experimental Materials}

Euglena gracilis was provided by the Institute of Freshwater Aquatic Sciences at the Chinese Academy of Sciences. The culture medium was prepared using deionized water. Hutner's Modified Medium(HUT) (Table 1) was prepared at a pressure of $1.05 \mathrm{~kg} / \mathrm{cm}^{2}$ and sterilized at $121.3^{\circ} \mathrm{C}$ for $20 \mathrm{~min}$ before use. The culture temperature was $25 \pm 1^{\circ} \mathrm{C}$, the illumination was 60 $\mu \mathrm{mol} /\left(\mathrm{m}^{2} \cdot \mathrm{s}\right)$, and the light-dark ratio was $12 \mathrm{~L}: 12 \mathrm{D}$ (light group) and 0 L:24 D (dark group). Cultivation flasks (500 $\mathrm{mL}$ ) were agitated several times each day to prevent microalgae from adhering to the walls or sedimenting.

\section{Experimental Methods}

\section{Analysis of Fatty Acid Composition}

For methyl esterification, samples were added to a $15 \mathrm{~mL}$ centrifuge tube and $2 \mathrm{~mL}$ of $2 \%$ sodium hydroxide in methanol was added. The mixture was flushed until oil droplets disappeared. Subsequently, $3 \mathrm{~mL}$ of $14 \%$ boron trifluoride in methanol was added, and the mixture was boiled for $30 \mathrm{~min}$. An appropriate amount of isooctane solution was added, the condenser was removed, and $20 \mathrm{~mL}$ of saturated sodium chloride solution was added. One to two milliliters of the upper layer of the solution was collected by pipetting, the sample was dehydrated by addition of anhydrous sodium sulfate, and the sample was injected (Chen \& Johns, 1996).

The analysis was performed using an Agilent $7890 \mathrm{~A}$ gas chromatograph-mass spectrometer equipped with a CNW CD-2560 chromatography column with a size of $100 \mathrm{~m} \times 0.25 \mathrm{~mm} \times 0.20 \mu \mathrm{m}$. The operating parameters were: inlet temperature, $250^{\circ} \mathrm{C}$; detector type, FID; detection temperature, $260^{\circ} \mathrm{C}$; injection volume, $1 \mu \mathrm{L}$;

Table 1. Huter's Modified Medium(HUT)

\begin{tabular}{lc}
\hline Component & Amount \\
\hline $\mathrm{KH}_{2} \mathrm{PO}_{4}$ & $0.02 \mathrm{~g}$ \\
$\mathrm{MgSO}_{4} \cdot 7 \mathrm{H}_{2} \mathrm{O}$ & $0.025 \mathrm{~g}$ \\
Yeast extract & $0.4 \mathrm{~g}$ \\
Sodium acetate & $0.4 \mathrm{~g}$ \\
peptone & $0.6 \mathrm{~g}$ \\
Deionized water & $1000 \mathrm{~mL}$ \\
\hline
\end{tabular}


split ratio, 10:1; carrier gas flow rate, $0.5 \mathrm{~mL} \cdot \mathrm{min}-1$. The temperature program used was $130^{\circ} \mathrm{C}$ for $5 \mathrm{~min}$, after which the temperature was increased to $240^{\circ} \mathrm{C}$ at a rate of a $4^{\circ} \mathrm{C}$ per min. The column was then maintained at $240^{\circ} \mathrm{C}$ for $30 \mathrm{~min}$.

\section{Preparation of Sequencing Samples}

After 5 days of culture, E. gracilis was centrifuged at $4^{\circ} \mathrm{C}(2000 \mathrm{r} / \mathrm{min}, 10 \mathrm{~min})$, and the supernatant was discarded. The sample was immediately frozen in liquid nitrogen $\left(-196^{\circ} \mathrm{C}\right)$ until RNA isolation (Zhao $\mathrm{W}$, et al. 2017). Total RNA was extracted using a Total RNA Extractor (Trizol, Sangon Biotech) according to the manufacturer's instructions. The quality of RNA samples was verified by NovogeneBioinformatics Technology Co., Ltd. (Beijing, China). The RNA was then enriched using magnetic beads containing oligo(dT). Fragmentation buffer was added to break the mRNA into short fragments, which served as templates to synthesize single-stranded cDNA using random hexamers. Buffer, dNTPs, DNA polymerase I, and RNase $H$ were then added for the synthesis of double-stranded cDNA, which was later purified using AMPure XP beads. The purified double-stranded cDNA was first subjected to end-repair, A-tailing, and adapter ligation, followed by size selection of the fragments using AMPure XP beads. Finally, polymerase chain reaction (PCR) amplification was performed, the products were purified using AMPure XP beads and a final library was obtained.

Preliminary quantification of the constructed library was performed using Qubit2.0. The library was diluted to $1.5 \mathrm{ng} / \mu \mathrm{L}$, and the insert size of the library was determined using the Agilent 2100 system. After the expected insert size was obtained, accurate quantification of the effective concentration of the library was performed using quantitative PCR (valid effective concentration $>2 \mathrm{nM}$ ) to ensure the quality of the library. After controlling for quality, the library was subjected to sequencing.

\section{Data Processing and De Novo Assembly}

The raw reads obtained from sequencing were preprocessed. Bases with linkers, low-quality bases, and uncertain bases were discarded to yield clean reads (The base number of Qphred <= 20 was more than $50 \%$ of the reads). Trinity (Grabherr et al., 2011) was used for assembly of the clean reads, yielding unigenes for the samples.

\section{Functional Annotation and Metabolic Pathway Analysis}

The unigenes obtained from assembly were compared to the Nr, Nt, Pfam (http://pfam.sanger.ac.uk/), KOG/COG (http://www.ncbi.nlm.nih.gov/COG/), SWISS-PROT (http://www.ebi.ac.uk/uniprot/), KEGG (http:///www.genome.jp/kegg/), and GO (http://www.geneontology.org/) databases. The EC numbers of the KEGG-annotated unigenes involved in the metabolic pathway were recorded.

Finally, the PKFM values in fatty acid biosynthesis, lipid degradation, and glycerolipid metabolism pathways for each unigene under different conditions were calculated to compare the expression levels of metabolic pathway genes.

\section{Results}

Change in E. gracilis Fatty Acid Content Under Light Stress

Table 2 lists the fatty acid content of $E$. gracilis under light and dark culture conditions. The fatty acid carbon chain composition of $E$. gracilis was between C12-C22 in the light group and mainly contained methyl

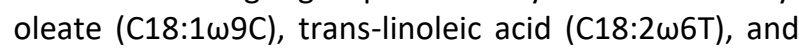
$\alpha$-linoleic acid (C18:3w3), which accounted for over $60 \%$ of all fatty acids. There were 19 fatty acid components in the light group, with the remainder comprising of 5

Table 2. Fatty acid content and composition of $E$. gracilis

\begin{tabular}{|c|c|c|c|c|c|}
\hline $\begin{array}{l}\text { Type of } \\
\text { fatty acid }\end{array}$ & $\begin{array}{l}\text { Light group } \\
\text { (g/100 g) }\end{array}$ & $\begin{array}{l}\text { Dark group } \\
(\mathrm{g} / 100 \mathrm{~g})\end{array}$ & Type of fatty acid & Light group (g/100 g) & Dark group (g/100 g) \\
\hline C8:0 & - & $0.002 \pm 0.000$ & C17:1 & $0.002 \pm 0.000$ & $0.011 \pm 0.001$ \\
\hline C10:0 & - & $0.031 \pm 0.001$ & $C 18: 1 \omega 9 C$ & $0.300 \pm 0.021$ & $0.170 \pm 0.001$ \\
\hline C11:0 & - & $0.003 \pm 0.000$ & 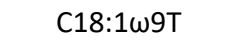 & $0.006 \pm 0.001$ & $0.002 \pm 0.000$ \\
\hline C12:0 & $0.051 \pm 0.002$ & $0.125 \pm 0.001$ & $\mathrm{C} 22: 1 \omega 9$ & - & $0.0002 \pm 0.000$ \\
\hline C13:0 & - & $0.046 \pm 0.001$ & C24:1 & - & $0.0001 \pm 0.000$ \\
\hline C14:0 & $0.016 \pm 0.001$ & $0.347 \pm 0.001$ & $C 18: 2 \omega 6 C$ & $0.111 \pm 0.018$ & $0.065 \pm 0.001$ \\
\hline C15:0 & $0.002 \pm 0.000$ & $0.040 \pm 0.001$ & $\mathrm{C} 18: 2 \omega 6 \mathrm{~T}$ & $0.273 \pm 0005$ & - \\
\hline C16:0 & $0.051 \pm 0.002$ & $0.324 \pm 0.003$ & $\mathrm{C} 18: 3 \omega 3$ & $0.513 \pm 0.003$ & $0.021 \pm 0.001$ \\
\hline C17:0 & - & $0.014 \pm 0.001$ & $\mathrm{C} 18: 3 \omega 6$ & $0.002 \pm 0.000$ & $0.006 \pm 0.000$ \\
\hline C18:0 & $0.007 \pm 0.001$ & $0.061 \pm 0.001$ & $\mathrm{C} 20: 2$ & $0.009 \pm 0.002$ & $0.102 \pm 0.001$ \\
\hline C20:0 & - & $0.002 \pm 0.000$ & $C 20: 3 \omega 3$ & $0.025 \pm 0.002$ & $0.035 \pm 0.001$ \\
\hline C24:0 & - & $0.013 \pm 0.000$ & $C 20: 3 \omega 6$ & - & $0.053 \pm 0.001$ \\
\hline C14:1 & - & $0.051 \pm 0.000$ & $C 20: 4 \omega 6$ & $0.010 \pm 0.000$ & $0.175 \pm 0.001$ \\
\hline C15:1 & $0.055 \pm 0.005$ & $0.001 \pm 0.000$ & $C 20: 5 \omega 3$ & $0.085 \pm 0.002$ & $0.175 \pm 0.002$ \\
\hline C16:1 & $0.013 \pm 0.001$ & $0.147 \pm 0.001$ & $C 22: 6 \omega 3$ & $0.045 \pm 0.003$ & $0.023 \pm 0.001$ \\
\hline
\end{tabular}


types of SFA, 5 types of MUFA, and 9 types of PUFA. The 5 types of saturated fatty acids mainly are lauric acid (C12:0), myristic acid (C14:0), pentadecanoic acid (C15:0), palmitic acid (C16:0), and octadecanoic acid (C18:0). Compared with the dark group, they had reduced content of (C8:0), (C10:0), (C11:0), (C13:0), (C17:0), (C20:0) and (C24:0).

The fatty acid carbon chain composition of $E$. gracilis in the dark group was between C8-C22. The dark group contained 29 fatty acid components, mainly myristic acid (C14:0), palmitic acid (C16:0), oleic acid

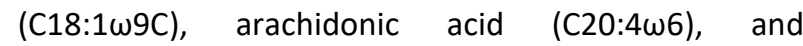
eicosapentaenoic acid (EPA) (C20: 5 accounted for over $58 \%$ of the total content, with the remainder composed of 12 types of SFA, 8 types of MUFA, and 9 types of PUFA.

Although the composition of fatty acid carbon chains in the light and dark groups was similar, the differences in fatty acid content and their relative proportions were very obvious. The total fatty acid content in the light and dark groups was $1.576 \mathrm{~g} / 100 \mathrm{~g}$ and $2.042 \mathrm{~g} / 100 \mathrm{~g}$, respectively. Figure 1 shows that the fatty acid content was higher in the dark than in the light group. The dark group also had the highest proportion of saturated fatty acids, at $49.29 \%$. The light group had the highest proportion of polyunsaturated fatty acids, at $68.08 \%$, while the proportion of SFA was only $8.05 \%$.
A

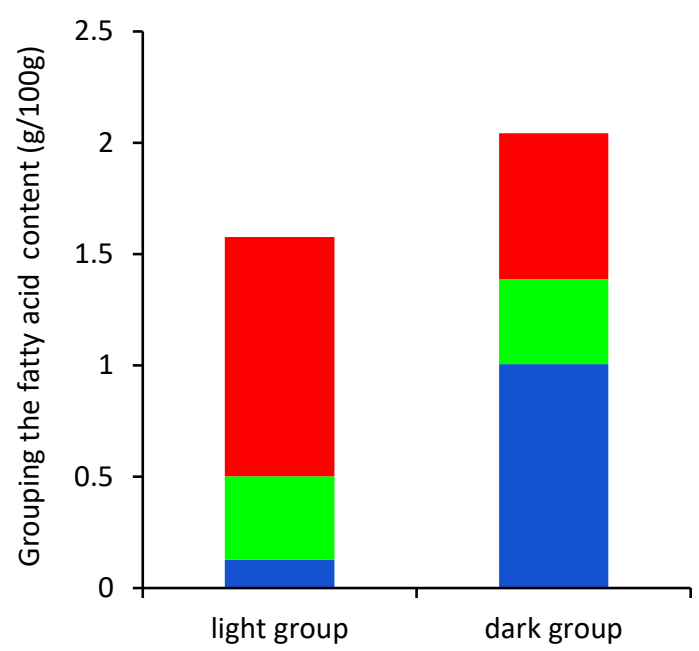

B

$\square$ SFA MUFA $\square$ PUFA

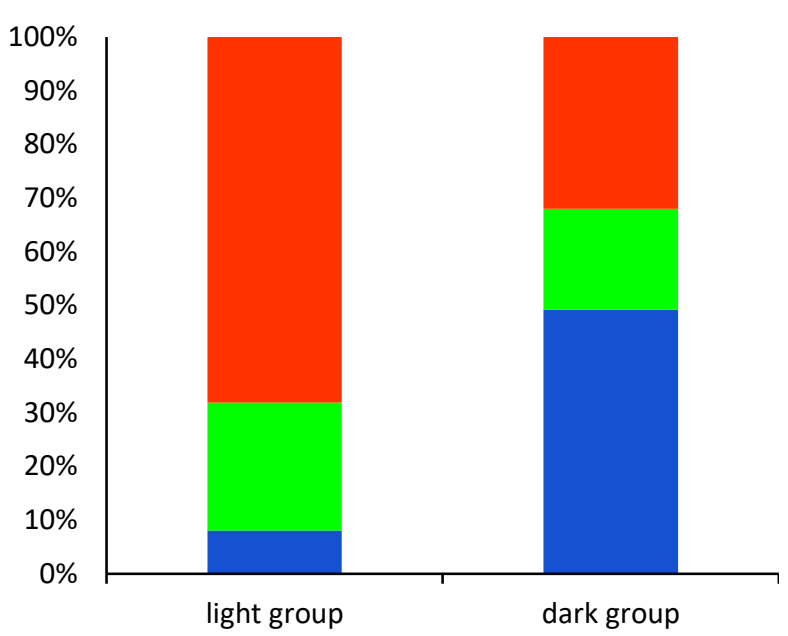

Figure 1. Fatty acid content and composition of E. gracilis grown under conditions of light or darkness. A: Fatty acid content grouped according to mass, B: Fatty acids grouped by percentage composition.

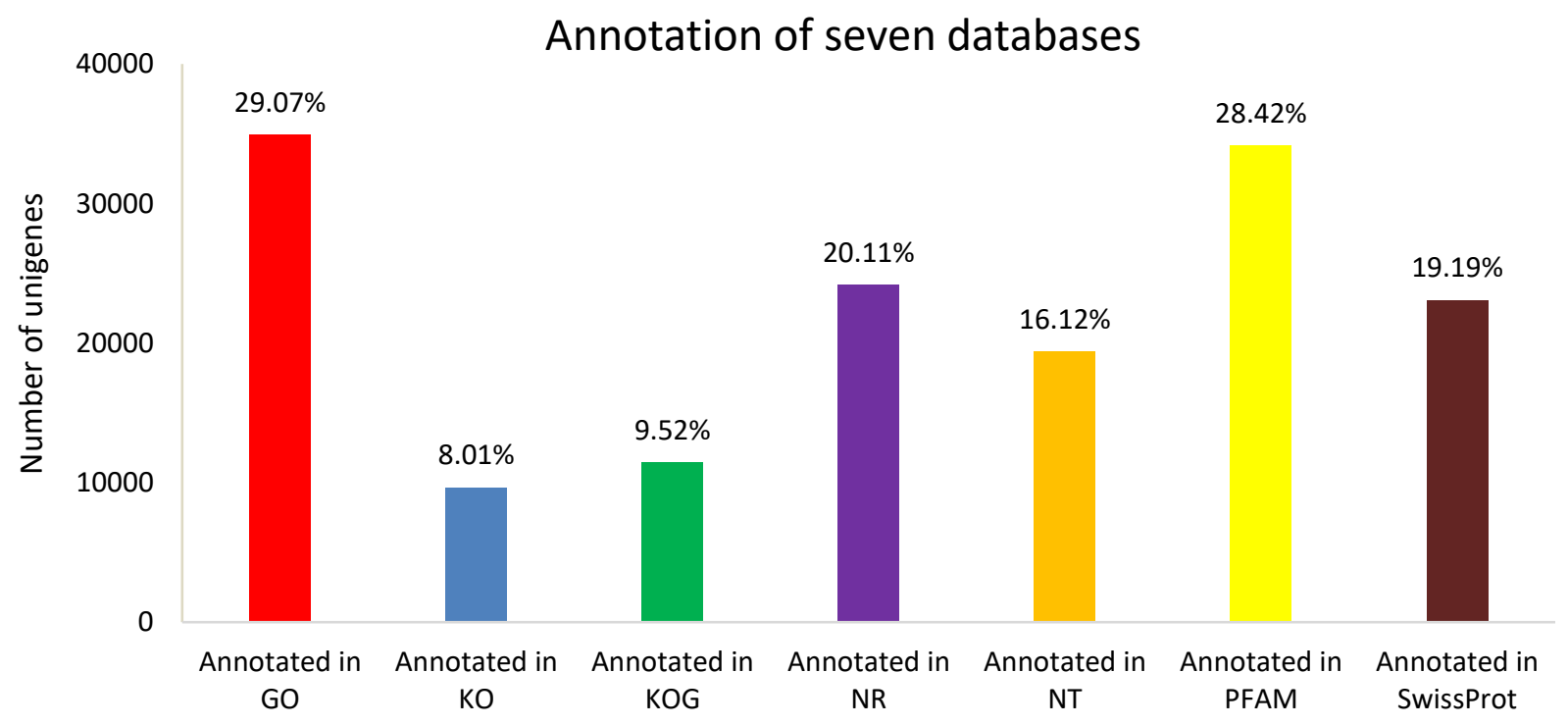

Figure 2. Annotation results for $E$. gracilis unigenes according to seven major databases 


\section{Transcriptome Sequencing Data and De Novo Assembly}

To understand the genes and metabolic pathways of enzymes associated with lipid synthesis in E. gracilis, algae in the light and dark groups were cultured for 5 days and then RNA was extracted. Illumina HiSeq 4000 paired-end sequencing was performed and 290,820,100 raw reads were obtained. After processing, 277,749,398 clean reads were obtained for de novo assembly, which accounted for $95.51 \%$ of raw reads. The clean reads were assembled using Trinity, and 297,648 transcripts and 120,086 unigenes were obtained, with an average length of $636 \mathrm{bp}$.

\section{Functional Annotation}

The unigenes obtained were subjected to functional annotation using seven major databases, with the results shown in Figure 2. The GO database had the most annotated unigenes at 34,915, accounting for $29.07 \%$ of all unigenes, followed by the PFAM database, with annotations for $28.42 \%$, KOG database, with annotations of 11436 transcripts (accounting for $9.52 \%$ ), and the KEGG database, with annotations of 9630 transcripts, accounting for $8.01 \%$.

\section{Metabolic Pathway Annotation and Lipid Metabolic Pathway Construction}

One hundred twenty-eight KEGG pathways were annotated from 9630 unigenes, including pathways for fatty acid biosynthesis, fatty acid degradation, glycerolipid metabolism, starch, and sucrose metabolism.

\section{Fatty Acid Biosynthesis}

The $E$. gracilis transcriptome contains transcripts of enzymes involved in fatty acid biosynthesis, as shown in Table 3. Based on the functional annotation of the $E$. gracilis transcriptome, a free fatty acid biosynthesis pathway was constructed in conjunction with the KEGG annotation results (Figure 3 ). Fatty acid synthesis occurs in the cytosol, and requires the transport of acetyl-CoA from the mitochondria to the vacuole, after which acetyl-CoA carboxylase (EC: 6.4.1.2 6.3.4.14) catalyzes the reaction of acetyl-CoA and carbonate to form malonyl-CoA, which is transformed into malonyl-ACP under the action of malonyl-CoA:acyl carrier protein transacylase (EC: 2.3.1.39). Acetyl-CoA carboxylase (EC: 6.4.1.2 6.3.4.14) is the rate-limiting enzyme in this pathway and is essential for de novo lipid synthesis and the synthesis of various fatty acids.

Figure $3 \mathrm{~B}$ shows the fatty acid carbon chain extension reaction, where malonyl-ACP serves as a carbon donor, adding two carbon-synthesized butyrylACPs to the carbon skeleton in the condensation reaction, 3-ketoacyl-ACP synthase II (EC: 2.3.1.179), 3ketoacyl ACP reductase (EC: 1.1.1.100), 3-hydroxyacylACP dehydratase (EC: 4.2.1.59), and enoyl-ACP reductase catalyze fatty acid condensation, reduction, dehydration, and re-reduction processes, respectively. 3-ketoacyl-ACP synthase II (EC: 2.3.1.179) catalyzes the initial condensation reaction and is an essential enzyme in the carbon chain extension process.

Under the catalysis by 3-ketoacyl-ACP synthase II (EC: 2.3.1.179), malonyl-ACP is converted into $\beta$ ketoacyl-ACP (3-oxooctadecanoyl-ACP), which is reduced under catalysis by 3-ketoacyl-ACP reductase (EC: 1.1.1.100), dehydrated under catalysis by 3hydroxyacyl-ACP dehydratase (EC: 4.2.1.59), and reduced under catalysis of enoyl ACP reductase (EC: 1.3.1.9 1.3.1.10) into a saturated fatty acid-ACP. At the same time, saturated fatty acid-ACP is produced under the catalysis by 3-ketoacyl-ACP synthase II (EC: 2.3.1.179), after which $\delta-9$ acyl-ACP desaturase (EC: 1.14.19.2) introduces a double bond into acyl-ACP, forming an unsaturated fatty acid-ACP. The saturated and unsaturated fatty acid-ACPs are finally converted into saturated and unsaturated fatty acids, respectively, under the action of oleoyl-ACP hydrolase (EC: 3.1.2.14).

\section{Fatty Acid Degradation}

The $E$. gracilis transcriptome contains transcripts of enzymes involved in fatty acid catabolism pathways, as shown in Table 4. Under the catalysis by acyl-CoA

Table 3. Enzymes identified in the fatty acid biosynthesis in E. gracilis

\begin{tabular}{|c|c|c|c|}
\hline KO name & Name & Unigene number & EC number \\
\hline K01897 & long-chain acyl-CoA synthetase & 27 & 6.2 .1 .3 \\
\hline K11262 & acetyl-CoA carboxylase / biotin carboxylase 1 & 10 & 6.4.1.2 6.3.4.14 \\
\hline K00059 & 3-oxoacyl-[acyl-carrier protein] reductase & 9 & 1.1.1.100 \\
\hline K03921 & acyl-[acyl-carrier-protein] desaturase & 7 & 1.14.19.2 \\
\hline K09458 & 3-oxoacyl-[acyl-carrier-protein] synthase II & 6 & 2.3.1.179 \\
\hline K10781 & fatty acyl-ACP thioesterase B & 3 & 3.1.2.14 3.1.2.21 \\
\hline K00645 & - & 3 & 2.3.1.39 \\
\hline K02372 & 3-hydroxyacyl-[acyl-carrier-protein] dehydratase & 2 & 4.2.1.59 \\
\hline K00208 & enoyl-[acyl-carrier protein] reductase I & 2 & 1.3.1.9 1.3.1.10 \\
\hline K01961 & acetyl-CoA carboxylase, biotin carboxylase subunit & 1 & 6.4.1.2 6.3.4.14 \\
\hline K10782 & fatty acyl-ACP thioesterase $A$ & 1 & 3.1.2.14 \\
\hline
\end{tabular}




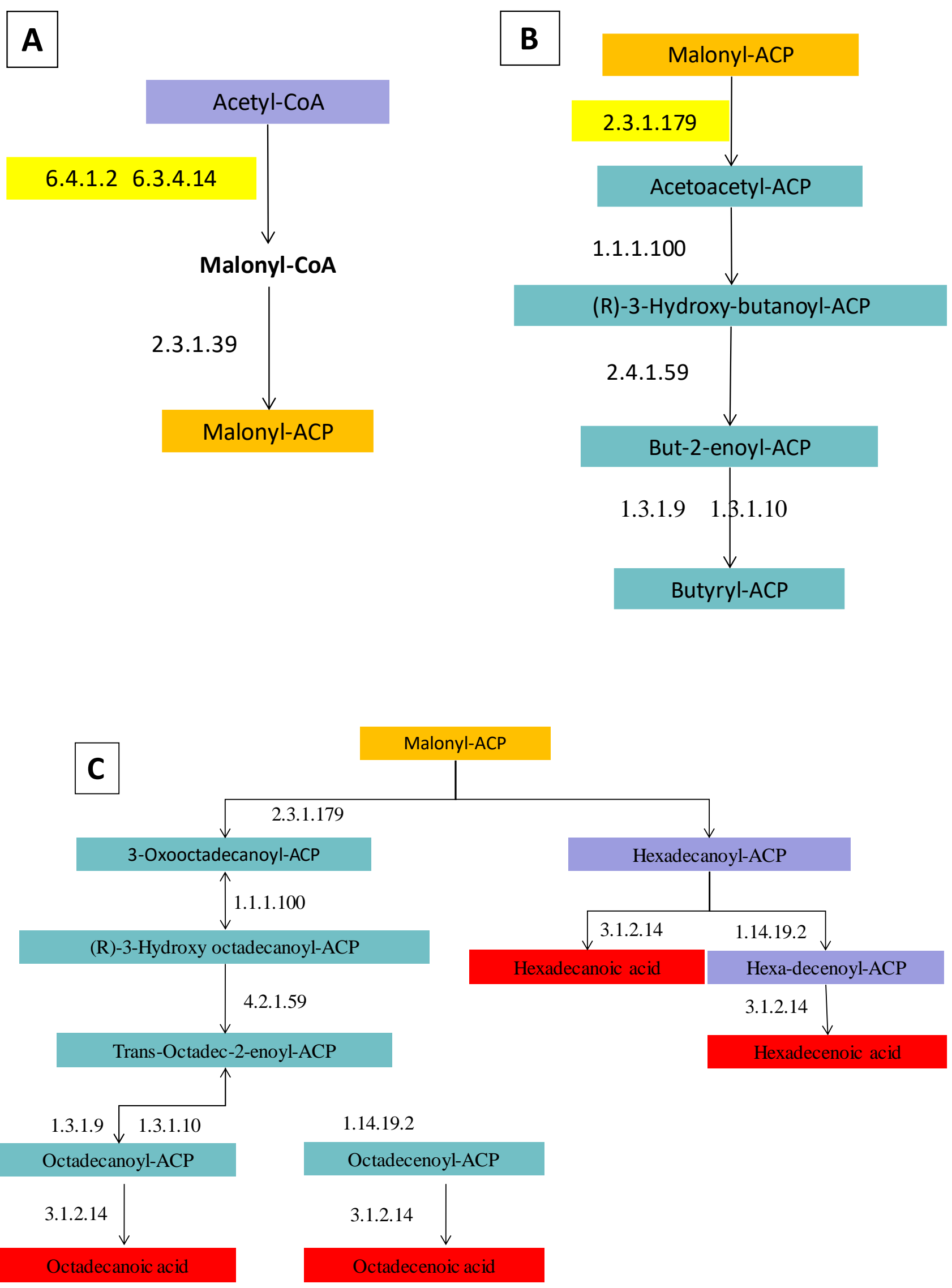

Figure 3. Fatty acid biosynthesis pathway reconstructed on the basis of de novo assembly and annotation of the $E$. gracilis transcriptome. A: Biosynthesis of malonyl-ACP, B: Biosynthesis of butyryl-ACP, C: Biosynthesis of fatty acids. 
synthetase (EC: 6.2.1.3), fatty acids are converted into hexa-decanoyl-CoA, which then enters the $\beta$-oxidation pathway. Two carbons are removed from hexadecanoyl-CoA through dehydrogenation, hydration, reduction, and thiolysis by ester acyl-CoA oxidase (EC: 1.3.3.6), enoyl-CoA hydratase (EC: 4.2.1.17), 3hydroxyalkyl-CoA dehydrogenase (EC: 4.2.1.17 1.1.1.35 1.1.1.211), and acetyl-CoA acyltransferase 1 (EC: 2.3 . 1.16), respectively, forming acetyl-CoA, which enters the tricarboxylic acid cycle, eventually being degraded into $\mathrm{H}_{2} \mathrm{O}$ and $\mathrm{CO}_{2}$ (Figure 4).

\section{Glycerolipid Metabolism}

The $E$. gracilis transcriptome contains transcripts of enzymes involved in Glycerolipid metabolism, as shown in Table 5. Triacylglycerol synthesis and degradation pathways were constructed based on KEGG cluster analysis of $E$. gracilis (Figure $5 \mathrm{~A}$ ). Glycerol kinase (EC: 2.7.1.30) catalyzes the conversion of glycerol into snglycerol-3-phosphate which, along with acyl-CoA, is a precursor for triacylglycerol synthesis. Glycerol-3phosphate O-acyltransferase (EC: 2.3.1.15) catalyzes

Table 4. Enzymes involved in fatty acid degradation in E. gracilis

\begin{tabular}{|c|c|c|c|}
\hline KO name & Name & Unigene number & EC number \\
\hline K00128 & aldehyde dehydrogenase (NAD+) & 29 & 1.2.1.3 \\
\hline K01897 & long-chain acyl-CoA synthetase & 27 & 6.2.1.3 \\
\hline K00626 & acetyl-CoA C-acetyltransferase & 9 & 2.3.1.9 \\
\hline K10527 & enoyl-CoA hydratase/3-hydroxyacyl-CoA dehydrogenase & 7 & $\begin{array}{l}\text { 4.2.1.17 1.1.1.35 } \\
\quad 1.1 \cdot 1.211\end{array}$ \\
\hline K00121 & $\begin{array}{l}\text { S-(hydroxymethyl)glutathione dehydrogenase / alcohol } \\
\text { dehydrogenase }\end{array}$ & 4 & 1.1.1.284 1.1.1.1 \\
\hline K07513 & acetyl-CoA acyltransferase 1 & 4 & 2.3.1.16 \\
\hline K18857 & alcohol dehydrogenase class-P & 3 & 1.1.1.1 \\
\hline K00232 & acyl-CoA oxidase & 3 & 1.3.3.6 \\
\hline K07511 & enoyl-CoA hydratase & 3 & 4.2.1.17 \\
\hline K00249 & acyl-CoA dehydrogenase & 2 & 1.3.8.7 \\
\hline K14085 & aldehyde dehydrogenase family 7 member $\mathrm{A} 1$ & 1 & 1.2.1.31 1.2.1.8 1.2.1.3 \\
\hline
\end{tabular}

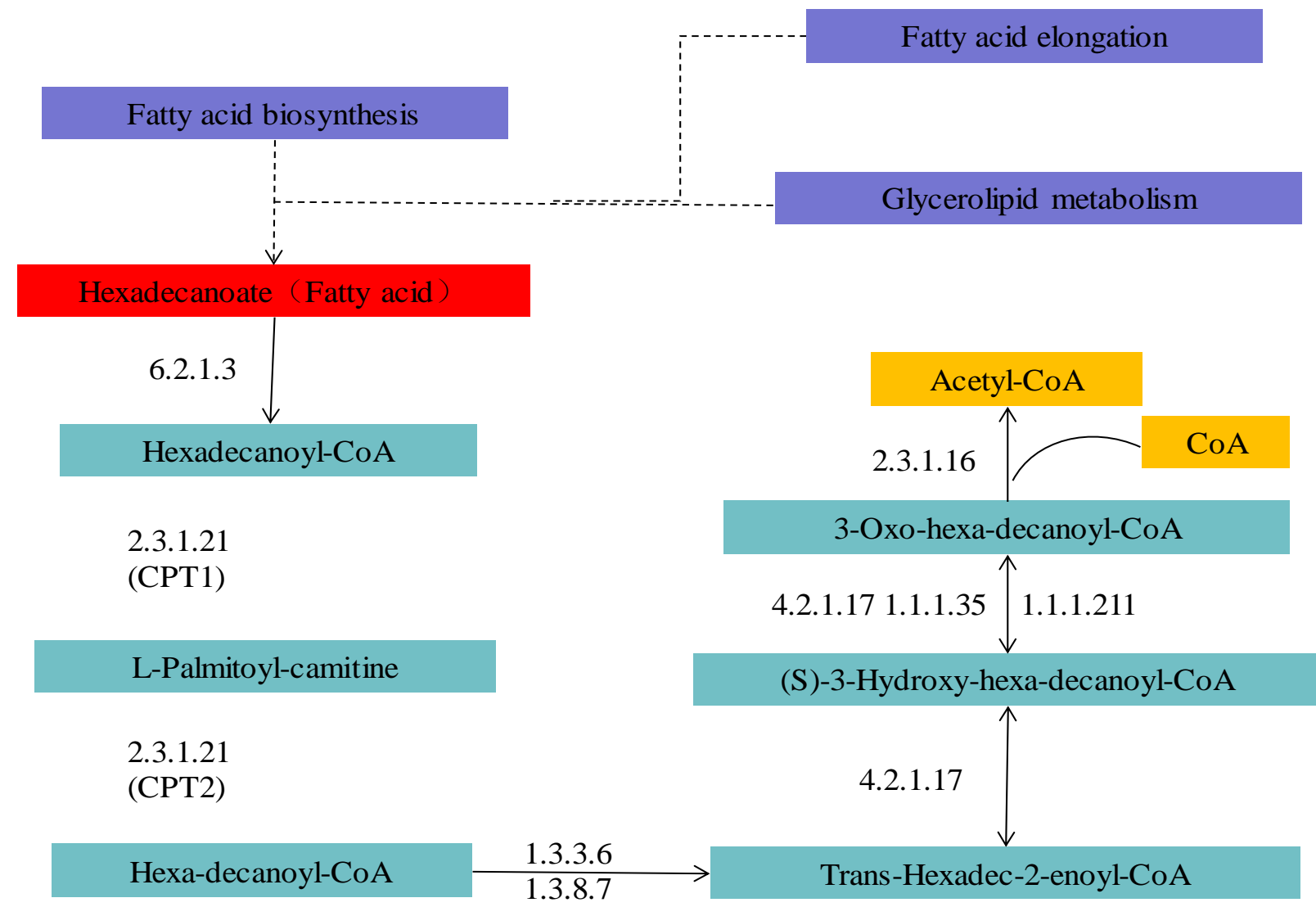

Figure 4. Fatty acid degradation pathway reconstructed on the de basis of de novo assembly and annotation of the $E$. gracilis. transcriptome 
esterification of the 1-carbon, forming 1-acyl-snglycerol-3-phosphate. 1-acyl-sn-glycero-3-phosphate acyltransferase catalyzes the esterification of the 2carbon, forming 1,2-diacyl-sn-glycerol-3-phosphate. Phosphatidate phosphatase (EC: 3.1.3.4) then catalyzes phosphorylation of 1,2-diacyl-sn-glycerol-3-phosphate into diacylglycerol. Phospholipid:diacylglycerol acyltransferase (EC: 2.3.1.158) catalyzes the esterification reaction that follows to form triacylglycerol. Phospholipid:diacylglycerol acyltransferase (EC: 2.3.1.158) is the rate-limiting enzyme for the synthesis of stored lipids, and is an essential enzyme for triacylglycerol synthesis.

The triacylglycerol degradation pathway is shown in Figure 5B. In this process, triacylglycerol is deacylated, forming diacylglycerol, and fatty acids are re-degraded. Triacylglycerol and diacylglycerol are converted into diacylglycerol and free fatty acids, respectively, by triacylglycerol lipase (EC: 3.1.1.3).

\section{Preliminary Analysis of Differential Gene Expression in Lipid Metabolism-Associated Pathways}

The expression levels of fatty acid biosynthesis pathway genes were compared using FPKM (expected number of fragments per kilobase of transcript sequence per million base pairs sequenced) values
(Table 6). It was found that, among the 71 unigenes involved in fatty acid biosynthesis metabolic pathways, there were 10 differentially-expressed genes, of which 5 were upregulated and 5 were downregulated. All changes in differentially expressed genes were no more than 2-fold. The essential and rate-limiting enzyme in the fatty acid biosynthesis pathway (EC: 6.4.1.2 6.3.4.14) was upregulated 1.69-fold under dark conditions.

There were 92 unigenes annotated to fatty acid degradation pathways, including 12 differentiallyexpressed genes (Table 7), of which, 2 were upregulated, whereas 10 were downregulated. The downregulated genes included acyl-CoA synthetase (EC: 6.2.1.3) and acyl -CoA dehydrogenase (EC: 1.3.8.7). Downregulation indicates that fatty acid degradation pathways are inhibited under dark conditions.

A total of 81 unigenes were annotated to triacylglycerol metabolic pathways, including 13 differentially-expressed genes, of which 4 genes were upregulated and 9 genes were downregulated under dark conditions; all changes in differentially expressed genes were within 2-fold (Table 8). The upregulated genes arylamine $\mathrm{N}$-acetyltransferase (EC: 2.3.1.5) and phosphatidate phosphatase (EC: 3.1.3.4) are mainly involved in the triacylglycerol synthesis pathway, indicating that E. gracilis tends toward triacylglycerol accumulation under dark conditions.

Table 5. Enzymes identified in glycerolipid metabolism in E. gracilis

\begin{tabular}{cccc}
\hline KO name & Name & Unigene number & EC number \\
\hline K01897 & long-chain acyl-CoA synthetase & 27 & 6.2 .1 .3 \\
K11262 & acetyl-CoA carboxylase / biotin carboxylase 1 & 10 & 6.4 .1 .26 .3 .4 .14 \\
K00059 & 3-oxoacyl-[acyl-carrier protein] reductase & 9 & 1.1 .1 .100 \\
K03921 & acyl-[acyl-carrier-protein] desaturase & 7 & 1.14 .19 .2 \\
K09458 & 3-oxoacyl-[acyl-carrier-protein] synthase II & 6 & 2.3 .1 .179 \\
K10781 & fatty acyl-ACP thioesterase B & 3 & 3.1 .2 .143 .1 .2 .21 \\
K00645 & - & 3 & 2.3 .1 .39 \\
K02372 & 3-hydroxyacyl-[acyl-carrier-protein] dehydratase & 2 & 4.2 .1 .59 \\
K00208 & enoyl-[acyl-carrier protein] reductase I & 2 & 1.3 .1 .91 .3 .1 .10 \\
K01961 & acetyl-CoA carboxylase, biotin carboxylase subunit & 1 & 6.4 .1 .26 .3 .4 .14 \\
K10782 & fatty acyl-ACP thioesterase A & 1 & 3.1 .2 .14 \\
\hline
\end{tabular}

Table 6. Differentially expressed genes involved in the fatty acid biosynthesis pathway in E. gracilis

\begin{tabular}{cccccc}
\hline Unigene ID & KO id & EC & FPKM (light group) & FPKM (dark group) & Extent of change \\
\hline TRINITY_DN30844_c0_g1 & K00059 & 1.1 .1 .100 & 75.21 & 45.92 & -1.67 \\
TRINITY_DN33990_c0_g2 & K03921 & 1.14 .19 .2 & 269.65 & 212.14 & -1.27 \\
TRINITY_DN37603_c4_g1 & K09458 & 2.3 .1 .179 & 389.38 & 333.78 & -1.17 \\
TRINITY_DN33947_c0_g2 & K01897 & 6.2 .1 .3 & 152.98 & 135.07 & -1.13 \\
TRINITY_DN38551_c1_g2 & K01897 & 6.2 .1 .3 & 355.52 & 349.17 & -1.02 \\
TRINITY_DN40360_c1_g1 & K11262 & 6.4 .1 .26 .3 .4 .14 & 61.31 & 109.97 & +1.79 \\
TRINITY_DN41561_c0_g2 & K11262 & 6.4 .1 .26 .3 .4 .14 & 107.92 & 176.76 & +1.64 \\
TRINITY_DN35843_c0_g1 & K00059 & 1.1 .1 .100 & 91.56 & 134.91 & +1.47 \\
TRINITY_DN39253_c0_g1 & K01897 & 6.2 .1 .3 & 644.83 & 819.77 & +1.27 \\
TRINITY_DN33890_c1_g2 & K01897 & 6.2 .1 .3 & 191.73 & 244.26 & +1.27 \\
\hline
\end{tabular}




\section{Discussion}

Effect of Light Exposure on Fatty Acid Content in E. gracilis

Light is one of the most important factors affecting the growth of unicellular algae and the composition of their metabolites. Light can participate in signal transduction through a series of ferredoxin and thioredoxin proteins, stimulating acetyl-CoA carboxylase (ACCase), and regulating fatty acid synthesis (Kozaki, Kamada, Iguchi, \& Sasaki, 2000). In general, the lipid content of E. gracilis is much lower than that of oleogenic microalgal species such as Chlorella (30-57\% lipid) (Wang, Rischer, Eriksen, \& Wiebe, 2013) and Nannochloropsis (26-42\% lipid) (Millán-Oropeza \& Fernández-Linares, 2016), but $E$. gracilis contains a high percentage of unsaturated fatty acids (Wang, Seppänen-
Laakso, Rischer, \& Wiebe, 2018). In this study, 14 unsaturated fatty acids were found in the light group, whereas 29 were found in the dark group.

In the present study, the total amount of fatty acids in the dark group was 1.3-fold that in the light group. The dark group showed 7.9-fold higher accumulation of SFA than in the light group, whereas PUFA content was 1.6-fold lower that in the light group. Dark conditions favored the accumulation of EPA (C20:5 133$)$ and ARA

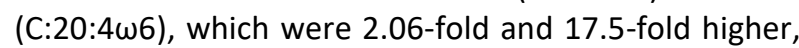
respectively, than in the light group. A study by Lee and Tan (1988) showed that the EPA content of Porphyridium cruentum increased as light intensity increased, which is contrary to the results of the present study. However, Cao, Sun, \& Mai (2010) found that ARA and PUFA content in green algae decreased as light intensity increased, consistent with the results of the present study. The observation that light leads to
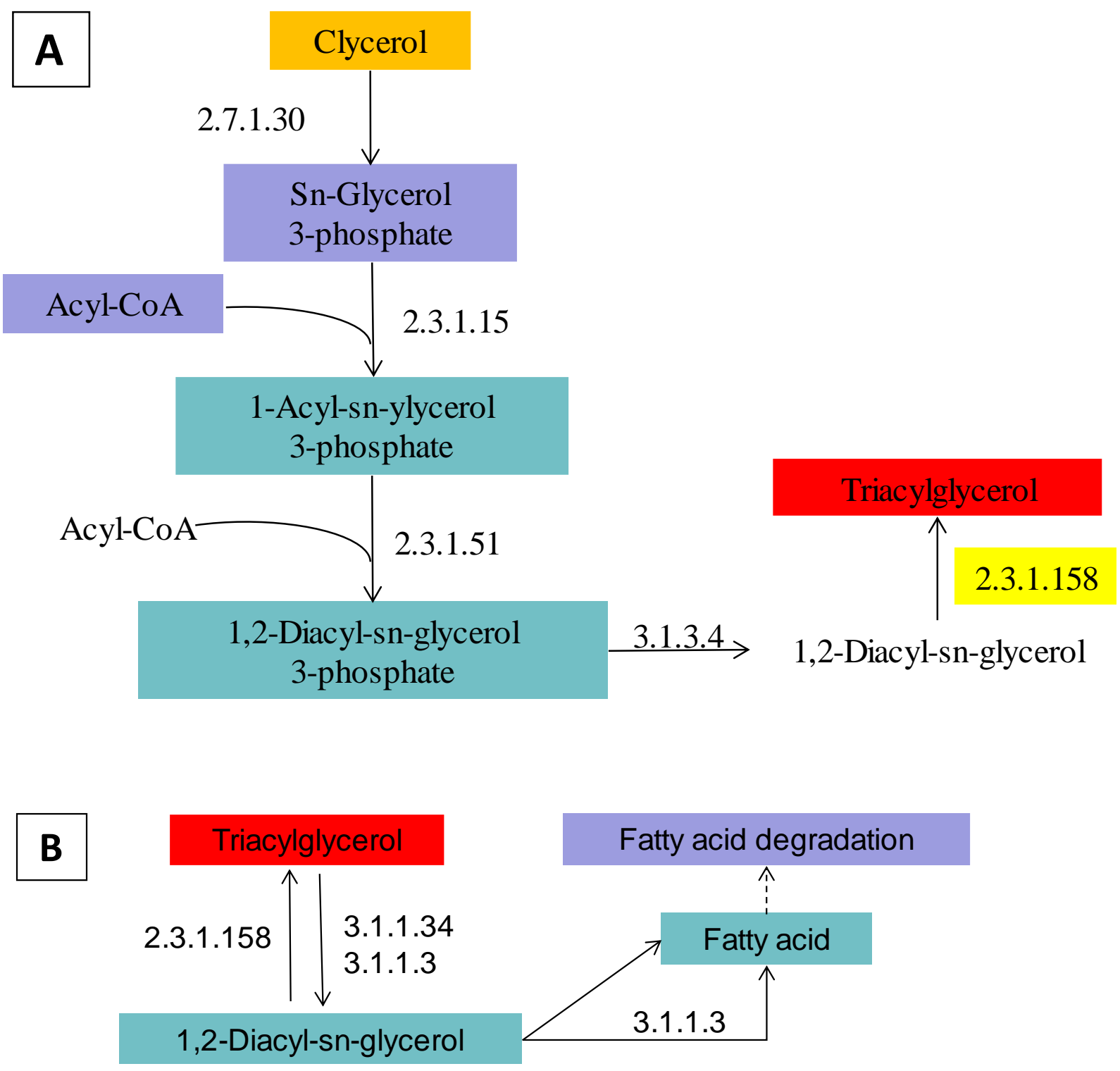

Figure 5. Glycerolipid metabolism pathway reconstructed on the basis of de novo assembly and annotation of E. gracilis transcriptome. A: Glycerolipid biosynthesis, B: Glycerolipid catabolism. 
different changes in fatty acid composition and content in microalgae could be species-related, a result of different types of desaturase present in the cells, or different effects of light intensity on enzymatic activity (Renaud et al., 1991). Another possibility is that the changes are a be the result of differences in light intensity used in the various experiments.

\section{Analysis of Transcriptome Data}

With the continued development of secondgeneration sequencing technologies, transcriptome sequencing has been applied in various fields. Currently, transcriptome sequencing has been used to study Dunaliella tertiolecta (Rismani-Yazdi, Haznedaroglu, Hsin, \& Peccia, 2012), Rhodomonas (Li et al., 2016), Chlorella minutissima (Yu, Yang, \& Lin, 2016), Thalassiosira pseudonana (Armbrust et al., 2004), and several other species. In addition, transcriptome data have been used to analyze genes coding for enzymes essential for some metabolic processes. In the present study, high-throughput sequencing technology was used to sequence the transcriptome of E. gracilis. After sequencing and assembly, 120,086 unigenes were obtained. A total of 48,031 unigenes were annotated using seven databases with an annotation rate of $39.99 \%$. This relatively low rate may be related to the relative lack of genetic sequence data for Euglena in public databases. It may also be that the unannotated genes represent genes unique to $E$. gracilis (Chen, $\mathrm{Li}$, Xiao, \& Liu, 2015).

\section{Analysis of Lipid-Associated Metabolic Pathways Lipid Biosynthesis and Degradation Pathways}

The unigenes were compared with those from the KEGG database, and 71 unigenes involved in fatty acid biosynthesis pathways, and 92 unigenes from catabolic pathways were screened. Using the resultant data, the fatty acid biosynthesis and degradation pathways of $E$. gracilis were constructed, and differential gene expression (FPKM) between the light and dark groups was analyzed.

Acetyl CoA carboxylase (ACCase, EC: 6.4.1.2 6.3.4.14) and 3-ketoacyl-ACP synthase II (EC: 2.3.1.179) are essential enzymes in the fatty acid biosynthesis pathway. Acetyl CoA carboxylase (ACCase, EC: 6.4 .1 .2 ) is the rate-limiting enzyme, and is essential for de novo lipid synthesis, and the synthesis of various fatty acids. ACCase is composed of a biotin carboxylase subunit, a biotin carboxyl carrier protein (BC-CP), and the $\alpha-C T$ and $\beta$-CT4 subunits of the two BCCP carboxyltransferases. Acetyl-CoA carboxylase (EC: 6.4.1.2 6.3.4.14) expression was upregulated 1.69-fold under dark conditions. 3-

Table 7. Differentially expressed genes involved in the fatty acid degradation pathway in $E$. gracilis

\begin{tabular}{lcccc}
\hline Unigene ID & EC & FPKM (light group) & FPKM (dark group) & Extent of change \\
\hline TRINITY_DN29885_c2_g2 & 2.3 .1 .9 & 2033.84 & 1450.84 & -1.41 \\
TRINITY_DN31507_c0_g2 & 1.2 .1 .3 & 59.99 & 45.48 & -1.32 \\
TRINITY_DN30652_c3_g2 & 1.2 .1 .3 & 15890.99 & 13009.2 & -1.22 \\
TRINITY_DN31275_c0_g2 & 1.3 .8 .7 & 229.63 & 187.75 & -1.22 \\
TRINITY_DN38249_c0_g2 & 2.3 .1 .9 & 334.01 & 277.25 & -1.20 \\
TRINITY_DN33947_c0_g2 & 6.2 .1 .3 & 152.98 & 135.07 & -1.13 \\
TRINITY_DN40906_c0_g3 & 1.1 .1 .2841 .1 .1 .1 & 146.34 & 141.65 & -1.03 \\
TRINITY_DN38551_c1_g2 & 6.2 .1 .3 & 355.52 & 349.17 & -1.02 \\
TRINITY_DN53848_C0_g1 & 1.2 .1 .3 & 3.6 & 0 & - \\
TRINITY_DN8615_c0_g1 & 1.2 .1 .3 & 2.56 & 0 & - \\
TRINITY_DN39253_c0_g1 & 6.2 .1 .3 & 644.83 & 819.77 & +1.27 \\
TRINITY_DN33890_c1_g2 & 1.2 .1 .3 & 191.73 & 244.26 & +1.27 \\
\hline
\end{tabular}

Table 8. Differentially expressed genes involved in the glycerolipid metabolism pathway in E. gracilis

\begin{tabular}{lccccc}
\hline Unigene ID & KO id & EC & FPKM (light group) & FPKM (dark group) & Extent of change \\
\hline TRINITY_DN35317_c0_g1 & K07407 & 3.2 .1 .22 & 100.73 & 53.7 & -1.88 \\
TRINITY_DN34620_c1_g1 & K14457 & 2.3 .1 .22 & 70.23 & 50.62 & -1.39 \\
TRINITY_DN31507_c0_g2 & K00128 & 1.2 .1 .3 & 59.99 & 45.48 & -1.32 \\
TRINITY_DN35334_c2_g3 & K13513 & $2.3 .1 .-2.3 .1 .51$ & 98.71 & 79.98 & -1.23 \\
TRINITY_DN30652_c3_g2 & K00128 & 1.2 .1 .3 & 15890.99 & 13009.2 & -1.22 \\
TRINITY_DN38913_c0_g2 & K00901 & 2.7 .1 .107 & 70.7 & 58.96 & -1.20 \\
TRINITY_DN36567_c1_g1 & K00655 & 2.3 .1 .51 & 327.99 & 211.77 & -1.54 \\
TRINITY_DN53848_c0_g1 & K00128 & 1.2 .1 .3 & 3.6 & 0 & - \\
TRINITY_DN8615_c0_g1 & K00128 & 1.2 .1 .3 & 2.56 & 0 & - \\
TRINITY_DN33712_c0_g1 & K00630 & 2.3 .1 .15 & 50.14 & 74.16 & +1.47 \\
TRINITY_DN38411_c2_g5 & K15728 & 3.1 .3 .4 & 24.32 & 32.11 & +1.32 \\
TRINITY_DN40234_c1_g1 & K06119 & $2.4 .1 .-$ & 49.66 & 64.59 & +1.30 \\
TRINITY_DN31198_C0_g1 & K15728 & 3.1 .3 .4 & 39.12 & 42.94 & +1.10 \\
\hline
\end{tabular}


ketoacyl-ACP synthase II (EC: 2.3.1.179) catalyzes the condensation reaction of a 16-carbon chain to an 18carbon chain and is an essential enzyme in the condensation reaction. While no 3-ketoacyl-ACP synthase I was found in the present study, this transcript was found in Eustigmatos cf. polyphem (Wan et al., 2012), Dunaliella tertiolecta (Rismani-Yazdi, Haznedaroglu, Bibby, \& Peccia, 2011), and Neochloris oleoabundans (Rismani-Yazdi, Haznedaroglu, Hsin, \& Peccia, 2012).

Under dark conditions, the rate-limiting enzyme in the fatty acid biosynthesis pathway, acetyl-CoA carboxylase (ACCase, EC: 6.4.1.2 6.3.4.14), was upregulated, while acyl-CoA synthetase (EC: 6.2.1.3) and acyl-CoA dehydrogenase (EC: 1.3.8.7) in the fatty acid degradation metabolic pathway was downregulated. This indicated that the increase in fatty acid content was due to the promotion of the fatty acid biosynthesis pathway and inhibition of the fatty acid degradation pathway, consistent with the increase in fatty acid content in dark conditions in the present study.

\section{Glycerolipid Metabolic Pathways}

A triacylglycerol metabolic pathway for $E$. gracilis was constructed using 81 unigenes. In addition, differential gene expression (FPKM) of these genes in the light and dark groups was analyzed.

In the present study, phospholipids acted as donor groups in triacylglycerol synthesis catalyzed by phospholipid:diacylglycerol acyltransferase (EC:2.3.1.258). This triacylglycerol synthesis pathway exists in some plants and yeast (Anders et al., 2000). A transcript encoding a phospholipid:diacylglycerol acyltransferase (EC: 2.3.1.258) was also found in the transcripts of Eustigmatos cf. polyphem (Wan et al., 2012) and Dunaliella tertiolecta (Rismani-Yazdi, Haznedaroglu, Bibby, \& Peccia, 2011). In addition to these pathways, another triacylglycerol synthesis pathway exists, in which 1,2-diacylglycerol is acylated at the 3-carbon to form triacylglycerol under the catalysis by diacylglycerol acyltransferase (EC: 2.3.1.20). However, no transcripts encoding this enzyme were found, and whether this pathway exists in E. gracilis remains to be determined.

Acetyl-CoA carboxylase (ACCase, EC: 6.4.1.2 6.3.4.14) and 3-ketoacyl-acylase ACP synthase II (EC: 2.3.1.179) are essential enzymes in fatty acid synthesis. Phospholipid: diacylglycerol acyltransferase (EC: 2.3.1.258) is a rate-limiting enzyme in the synthesis of stored lipids, and is essential for triacylglycerol synthesis. Regulation of its activity can increase the content and composition of fatty acids and triacylglycerols in E. gracilis (Padham et al., 2007; Kroon, Wenxue, Simon, \& Slabas, 2006; Wendel, Lewin, \& Coleman, 2009). Inhibition or knockout of enzymes associated with fatty acid and triacylglycerol degradation through genetic engineering could also increase fatty acid and triacylglycerol content in $E$. gracilis. In this regard, the most common genetic modification used to date is overexpression of ACCase (Roessler, Bleibaum, Thompson, \& Ohlrogge, 2010). In addition, modification of essential enzymes regulating the condensation reaction in fatty acid synthesis pathways (Verwoert, Linden, Walsh, Nijkamp, \& Stuitje, 1995) and blockade of $\beta$-oxidation (Radakovits, Jinkerson, Darzins, \& Posewitz, 2010) are also effective means of promoting fatty acid accumulation.

\section{Conclusion}

In the present study, transcripts encoding enzymes related to fatty acid synthesis in the $E$. gracilis transcriptome were identified, and the pathways they are involved in were described. We studied lipid metabolism in Euglena gracilis under conditions of light stress using transcriptome analysis to characterize the enzymes and pathways involved. We also analyzed the lipid composition and content under different light conditions using gas chromatography. We found that by regulating the activity of acetyl-CoA carboxylase, 3ketoacyl-ACP synthase II, and phospholipid:diacylglycero acyltransferase, enzymes associated with fatty acid and glycerolipid degradation may be inhibited or knocked out, thereby increasing fatty acid and glycerolipid content and composition in $E$. gracilis. Our findings can provide a clear guide for genetic engineering of $E$. gracilis and modification of its lipid metabolism pathways. Additionally, our study provides a basis for genetic modification of E. gracilis for the construction of high-quality oil-producing engineered strains.

\section{Acknowledgements}

This work was supported by the Tianjin Rural Working Committee (grant number ITTFRS2017005); the Tianjin Science and Technology Commission (grant number 18JCTPJC67400); the Tianjin Science and Technology Commission (grant number 18ZXYENC00070); the Tianjin Science and Technology Commission (grant number 17PTSYJC00170); National Key Laboratory of Satellite Ocean Environmental Dynamics Open Fund Project (grant number SOED1419); and the Tianjin Agricultural University Postgraduate Training Program (grant number 2017YPY031).

\section{References}

Afiukwa, C. A., \& Ogbonna, J. C. (2017). Effects of mixed substrates on growth and vitamin production by Euglena gracilis. African Journal of Biotechnology, 26(22), 26122615. http://dx.doi.org/10.5897/AJB2007.000-2417

Ahmadinejad, N., Dagan, T., \& Martin, W. (2007). Genome history in the symbiotic hybrid Euglena gracilis. Gene, 402(1), 35-39. doi: 10.1016/j.gene.2007.07.023 http://dx.doi.org/10.1016/j.gene.2007.07.023

Anders, D., Ulf, S., Marit, L., Antoni, B., Michael, L., Line, S., \& Sten, S. (2000). Phospholipid:diacylglycerol 
acyltransferase: an enzyme that catalyzes the acyl-coaindependent formation of triacylglycerol in yeast and plants. Proceedings of the National Academy of Sciences, 97(12), 6487-6492.

http://dx.doi.org/10.1073/pnas.120067297

Armbrust, E. V., Berges, J. A., Bowler, C., Green, B. R., Martinez, D., Putnam, N. H., \& Rokhsar, D. S. (2004). The genome of the diatom Thalassiosira pseudonana: ecology, evolution, and metabolism. Science, 79-86. http://dx.doi.org/10.1126/science.1101156

Cao, C., Sun, S., \& Mai, K. (2010). Effect of light intensity on the total lipid contents and fatty acid composition in 4 strains of marine green algae. Acta Ecologica Sinica, 30(9), 2347-2353.

http://dx.doi.org/10.1016/S1872-5813(11)60001-7

Chen, F. \& Johns, M. R. (1996). Relationship between substrate inhibition and maintenance energy of Chlamydomonas reinhardtii in heterotrophic culture. Journal of Applied Phycology, 8(1), 15-19. http://dx.doi.org/10.1007/BF02186216

Chen, X., Li, J., Xiao, S., \& Liu, X. (2015). De novo assembly and characterization of foot transcriptome and microsatellite marker development for paphia textile. Gene, 576(1 Pt 3), 537. http://dx.doi.org//10.1016/j.gene.2015.11.001

Coyle, E. D., \& Simmons, R. A. (2014). Understanding the Global Energy Crisis. http://dx.doi.org/10.26530/OAPEN_469619

Dehesh, K., Tai, H., Edwards, P., Byrne, J., \& Jaworski, J. G. (2001). Overexpression of 3-ketoacyl-acyl-carrier protein synthase IIIs in plants reduces the rate of lipid synthesis. Plant Physiology, 125(2), 1103-1114. http://dx.doi.org/10.1104/pp.125.2.1103

Grabherr, M. G., Haas, B. J., Yassour, M., Levin, J. Z., Thompson, D. A., Amit, I., Adiconis, X., Fan, L., Raychowdhury, R., Zeng, Q., Chen, Z., Mauceli, E., Hacohen, N., Gnirke, A., Rhind, N., Palma, F. D., Birren, B. W., Nusbaum, C., Lindblad-Toh, K., Friedman, N., \&Regev, A. (2011). Fulllength transcriptome assembly from RNA-Seq data without a reference genome. Nature Biotechnology, 29(7), 644. http://dx.doi.org/10.1038/nbt.1883

Hsieh, H. J., Su, C. H., \& Chien, L. J. (2012). Accumulation of lipid production in Chlorella minutissima by triacylglycerol biosynthesis-related genes cloned from Saccharomyces cerevisiae and Yarrowia lipolytica. Journal of Microbiology, 50(3), 526-534.

http://dx.doi.org/10.1007/s12275-012-2041-5

$\mathrm{Hu}, \mathrm{Q}$., Sommerfeld, M., Jarvis, E., Ghirardi, M., Posewitz, M. M, \& Darzins, A. (2010). Microalgal triacylglycerols as feedstocks for biofuel production: perspectives and advances. Plant Journal, 54(4), 621-639.

http://dx.doi.org/10.1111/j.1365-313X.2008.03492.x

Khozin-Goldberg, I., Bigogno, C., Shrestha, P., \& Cohen, Z. (2010). Nitrogen starvation induces the accumulation of arachidonic acid in the freshwater green alga Parietochloris Incisa (Trebuxiophyceae). Journal of Phycology, 38(5), 991-994. http://dx.doi.org/10.1046/j.1529-8817.2002.01160.x

Kozaki, A., Kamada, K., Y, Iguchi, H., \& Sasaki, Y. (2000). Recombinant carboxyltransferase responsive to redox of pea plastidic acetyl-CoA carboxylase. Journal of Biological Chemistry, 275(14), 10702-10708. http://dx.doi.org/10.1074/jbc.275.14.10702

Krajčovič, J., Vesteg, M., \& Schwartzbach, S. D. (2015). Euglenoid flagellates: A multifaceted biotechnology platform. Journal of Biotechnology, 202, 135-145. http://dx.doi.org/10.1016/j.jbiotec.2014.11.035

Kroon, J. T. M., Wenxue, W., Simon, W. J., \& Slabas, A. R. (2006). Identification and functional expression of a type 2 acyl-CoA:diacylglycerol acyltransferase (DGAT2) in developing castor bean seeds which has high homology to the major triglyceride biosynthetic enzyme of fungi and animals. Phytochemistry, 67(23), 2541-2549. http://dx.doi.org/10.1016/j.phytochem.2006.09.020

Lee, Y. K., \& Tan, H. M. (1988). Effect of temperature, light intensity and dilution rate on the cellular composition of red alga Porphyridium cruentum in light-limited chemostat cultures. Mircen Journal of Applied Microbiology \& Biotechnology, 4(2), 231-237. http://dx.doi.org/10.1007/BF01301953

Li, H., Wang, W., Wang, Z., Lin, X., Fang, Z., \& Yang, L. (2016). De novo transcriptome analysis of carotenoid and polyunsaturated fatty acid metabolism in Rhodomonas sp. Journal of Applied Phycology, 28(3), 1649-1656. http://dx.doi.org/10.1007/s10811-015-0703-5

Millán-Oropeza, A., \& Fernández-Linares, L. (2016). Biomass and lipid production from Nannochloropsis oculata growth in raceway ponds operated in sequential batch mode under greenhouse conditions. Environmental Science \& Pollution Research, 24(33), 1-9. http://dx.doi.org/10.1007/s11356-016-7013-6

Molony, T. (2011). Bioenergy policies in Africa: mainstreaming gender amid an increasing focus on biofuels. Biofuels Bioproducts \& Biorefining, 5(3), 330-341. http://dx.doi.org/10.1002/bbb.293

Ogbonna, J. C. (2009). Microbiological production of tocopherols: current state and prospects. Applied Microbiology \& Biotechnology, 84(2), 217-225. http://dx.doi.org/10.1007/s00253-009-2104-7

Padham, A. K., Hopkins, M. T., Wang, T.W., Mcnamara, L. M., Maisie, L., Richardson, L. G. L., Smith, M. D., Taylor, C. A., \& Thompson, J. E. (2007). Characterization of a plastid triacylglycerol lipase from arabidopsis. Plant Physiology, 143(3), 1372-1384. http://dx.doi.org/10.1104/pp.106.090811

Pulz, O., \& Gross, W. (2004). Valuable products from biotechnology of microalgae. Appl Microbiol Biotechnol, 65(6), 635-648. http://dx.doi.org/10.1007/s00253-004$1647-x$

Radakovits, R., Jinkerson, R. E., Darzins, A., \& Posewitz, M. C. (2010). Genetic engineering of algae for enhanced biofuel production. Eukaryotic Cell, 9(4), 486-501. http://dx.doi.org/10.1128/EC.00364-09

Rawisara, R., Supapon, C., \& Kobkul, L. (2009). Overexpression of acetyl-CoA carboxylase gene of Mucor rouxii enhanced fatty acid content in Hansenula polymorpha. Molecular Biotechnology, 42(3), 327-332. http://dx.doi.org/10.1007/s12033-009-9155-y

Renaud, S. M., Parry, D. L., Thinh, L. V., Kuo, C., Padovan, A., \& Sammy, N. (1991). Effect of light intensity on the proximate biochemical and fatty acid composition of Isochrysis sp. and Nannochloropsis oculata for use in tropical aquaculture. Journal of Applied Phycology, 3(1), 43-53. http://dx.doi.org/10.1007/BF00003918

Rismani-Yazdi, H., Haznedaroglu, B. Z., Bibby, K., \& Peccia, J. (2011). Transcriptome sequencing and annotation of the microalgae Dunaliella tertiolecta: Pathway description and gene discovery for production of next-generation biofuels. Bmc Genomics, 12(1), 148. http://dx.doi.org/10.1186/1471-2164-12-148 
Rismani-Yazdi, H., Haznedaroglu, B. Z., Hsin, C., \& Peccia, J. (2012). Transcriptomic analysis of the oleaginous microalga Neochloris oleoabundans reveals metabolic insights into triacylglyceride accumulation. Biotechnology for Biofuels, 5(1), 74-74.

http://dx.doi.org/10.1186/1754-6834-5-74

Roessler, P. G., Bleibaum, J. L., Thompson, G. A., \& Ohlrogge, J. B. (2010). Characteristics of the gene that encodes acetyl-CoA carboxylase in the diatom Cyclotella cryptica. Annals of the New York Academy of Sciences, 721(1), 250-256.

http://dx.doi.org/10.1111/j.1749-6632.1994.tb47398.x

Savage, N. (2011). Algae: The scum solution. Nature, 474(7352), S15-S16. http://dx.doi.org/10.1038/474S015a

Scott, S. A., Davey, M. P., Dennis, J. S., Horst, I., Howe, C. J., Lea-Smith, D. J., \& Smith, A. G. (2010). Biodiesel from algae: challenges and prospects. Current Opinion in Biotechnology, 21(3), 277-286. http://dx.doi.org/10.1016/j.copbio.2010.03.005

Ho, S. H., Chen, C. Y., Lee, D. J., \& Chang, J. S. (2010). Perspectives on microalgal $\mathrm{CO}_{2}$-emission mitigation systems--a review. Biotechnology Advances, 29(2), 189. http://dx.doi.org/10.1016/j.biotechadv.2010.11.001

Solovchenko, A. E., Khozin-Goldberg, I., Didi-Cohen, S., Cohen, Z., \& Merzlyak, M. N. (2008a). Effects of light and nitrogen starvation on the content and composition of carotenoids of the green microalga Parietochloris incisa. Russian Journal of Plant Physiology, 55(4), 455-462. http://dx.doi.org/10.1134/s1021443708040043

Solovchenko, A. E., Khozin-Goldberg, I., Didi-Cohen, S., Cohen, Z., \& Merzlyak, M. N. (2008b). Effects of light intensity and nitrogen starvation on growth, total fatty acids and arachidonic acid in the green microalga Parietochloris incisa. Journal of Applied Phycology, 20(3), 245-251. http://dx.doi.org/10.1007/s10811-007-9233-0

Sun, A., Hasan, M. T., Hobba, G., Nevalainen, H., \& Te'o, J. (2018). Comparative assessment of the Euglena gracilis var. saccharophila variant strain as a producer of the $\beta$ 1,3-glucan paramylon under varying light conditions. Journal of Phycology, 54(4), 529-538. http://dx.doi.org/10.1111/jpy.12758

Verwoert, I. I. G. S., Linden, K. H. V. D., Walsh, M. C., Nijkamp, H. J. J., \& Stuitje, A. R. (1995). Modification of Brassica napus seed oil by expression of the Escherichia coli fabH gene, encoding 3-ketoacyl-acyl carrier protein synthase III. Plant Molecular Biology, 27(5), 875-886.
http://dx.doi.org/10.1007/BF00037016

Wan, L. L., Han, J., Sang, M., Li, A. F., Wu, H., Yin, S. J., \& Zhang, C. W. (2012). Retraction: De novo transcriptomic analysis of an oleaginous microalga: pathway description and gene discovery for production of next-generation biofuels. Plos One, 7(6), e35142.

http://dx.doi.org/10.1371/annotation/3155a3e9-5fbe435c-a07a-e9a4846ec0b6

Wang, Y., Rischer, H., Eriksen, N. T., \& Wiebe, M. G. (2013). Mixotrophic continuous flow cultivation of Chlorella protothecoides for lipids. Bioresource Technology, 144(6), 608-614.

http://dx.doi.org/10.1016/j.biortech.2013.07.027

Wang, Y., Seppänen-Laakso, T., Rischer, H., \& Wiebe, M. G. (2018). Euglena gracilis growth and cell composition under different temperature, light and trophic conditions. Plos One, 13(4), e0195329-. http://dx.doi.org/10.1371/journal.pone.0195329

Wendel, A. A., Lewin, T. M., \& Coleman, R. A. (2009). Glycerol3-phosphate acyltransferases: Rate limiting enzymes of triacylglycerol biosynthesis. BBA - Molecular and Cell Biology of Lipids, 1791(6), 501-506. http://dx.doi.org/10.1016/j.bbalip.2008.10.010

Yu, M., Yang, S., \& Lin, X. (2016). De- novo assembly and characterization of Chlorella minutissima UTEX2341 transcriptome by paired-end sequencing and the identification of genes related to the biosynthesis of lipids for biodiesel. Marine Genomics, 25, 69-74. http://dx.doi.org/10.1016/j.margen.2015.11.005

Yusuf, C. (2007). Biodiesel from microalgae. Biotechnology Advances, 25(3), 294-306. http://dx.doi.org/10.1016/j.biotechadv.2007.02.001

Zhang, C. W., Cohen, Z., Khozin-Goldberg, I., \& Richmond, A. (2002). Characterization of growth and arachidonic acid production of Parietochloris incisa comb. nov (Trebouxiophyceae, Chlorophyta). Journal of Applied Phycology, 14(6), 453-460. http://dx.doi.org/10.1023/a:1022375110556

Zhao, W., Wang, L., Liu, M., Jiang, K., Wang, M., Yang, G., Qi, C., \& Wang, B. (2017). Transcriptome, antioxidant enzyme activity and histopathology analysis of hepatopancreas from the white shrimp, Litopenaeus vannamei, fed with aflatoxin B1(AFB1). Developmental \& Comparative Immunology, 74:69-81. http://dx.doi.org/10.1016/j.dci.2017.03.031 